\title{
Embolization of an Exophytic Posterior Neck Mass Secondary to a Cutaneous Renal Cell Carcinoma Metastasis
}

\author{
Dallas E. Kramer, BS ${ }^{1}$, Mena G. Kerolus, $\mathrm{MD}^{2}$, Lee A. Tan, $\mathrm{MD}^{3}$, Smita Patel, $\mathrm{MD}^{4}$, Vijaya Reddy, $\mathrm{MD}^{4}$, \\ Michael Chen, $\mathrm{MD}^{2}$ \\ ${ }^{1}$ Rush Medical College, Rush University Medical Center, Chicago, IL, USA \\ ${ }^{2}$ Department of Neurosurgery, Rush University Medical Center, Chicago, IL, USA \\ ${ }^{3}$ Department of Neurosurgery, University of California San Francisco, San Francisco, CA, USA \\ ${ }^{4}$ Department of Pathology, Rush University Medical Center, Chicago, IL, USA
}

Renal cell carcinoma (RCC) commonly metastasizes to the lung, liver, bones, and brain; however, cutaneous metastases remain rare with few reported cases. Since RCCs have the propensity to metastasize to highly vascular areas, the scalp and skin of the head and neck region are likely locations for cutaneous metastases. We report a rare case of a large, exophytic, cauliflower-like, hemorrhagic, metastatic mass of the posterior neck. This is the first reported case of a head and neck cutaneous RCC metastasis treated with endovascular embolization prior to surgical resection. Due to the increased vascularity of RCCs and risk of excessive hemorrhage during resection, adjunctive embolization of cutaneous head and neck metastasis may have a role. Essential characteristics to our treatment strategy are discussed with a review of pertinent literature.

Key Words: Cutaneous metastasis; Embolization; Exophytic mass; Neck mass; Carcinoma, renal cell

\section{INTRODUCTION}

Renal cell carcinomas (RCC) commonly metastasizes to the lung, liver, bones, and brain; however, cutaneous metastases remains rare with few reported cases. Since RCCs have the propensity to metastasize to highly vascular areas, the scalp and skin of the head and neck region are likely locations for cutaneous metastases. We report a rare case of a large, exophytic, cauliflower-like, hemorrhagic, metastatic mass of the posterior neck. This is the first reported case of a head and neck cutaneous RCC metastasis treated with endovascular embolization prior to surgical resection.
Essential characteristics to our treatment strategy are discussed with a review of pertinent literature.

\section{CASE REPORT}

A man in his 60 s presented to the emergency room with large, exophytic posterior neck mass continuous with a yellow-tan nodule in the subcutaneous tissue measuring a $5.7 \times 5.0 \mathrm{~cm}$ that was bleeding (Fig. 1A). The patient stated that this mass had grown significantly in size over a short period of time and would spontaneously bleed on his clothes and bedding. His medical his-

\author{
Correspondence to: \\ Dallas E. Kramer, BS \\ Rush Medical College, Rush University \\ Medical Center, 1725 West Harrison \\ Street, Chicago, IL 60612, USA \\ Tel: +1-312-9426644 \\ Fax: +1-312-9422176 \\ E-mail: dallas_e_kramer@rush.edu \\ Received: May 28, 2020 \\ Revised: July 6, 2020 \\ Accepted: July 8, 2020
}

pISSN 2093-9043 eISSN 2233-6273 
tory included metastatic RCC diagnosed four years ago for which he underwent a left nephrectomy and metastatic disease to the lumbar spine for which he also underwent surgical resection and radiation. A short course of chemotherapy was stopped because of poor response.

On examination, the neck mass was cauliflower-shaped, grey-tan in color, rubbery in consistency, with some bleeding originating from the necrotic center (Fig. 1A). The necrotic core was bleeding and temporary hemostasis was achieved. A magnetic resonance imaging (MRI) of the neck demonstrated an exophytic, cutaneous neck mass without involvement deep to the posterior cervical fascia (Fig. 1B). Digital subtraction angiography demonstrated primary feeders to be the bilateral occipital arteries (Fig. 1C, D). Additionally, selective catheterization of the bilateral thyrocervical and costocervical trunks demonstrated prominent ascending and deep cervical vessels and tumor blush. Arterial feeder supply was also noted from the muscular branches of the right V1 segment of the vertebral artery (VA). There was no tumor involvement of the left VA.

The patient was counseled about these findings and given the extensive arterial supply, prior spontaneous hemorrhage, the surgical team requested preoperative embolization prior to resection. Direct puncture of the lesion was considered given the long distance and tortuosity of the primary occipital artery feeders. However, the unusually large diameter of the occipital artery feeders and safer hemostatic control associated with a femoral puncture influenced us to proceed with a femoral approach transarterial occipital artery embolization.

The left external carotid feeders were selectively catheterized using a Scepter (MicroVention, Aliso Viejo, CA, USA) microcatheter over a Synchro 14 microwire (Stryker, Freemont, CA, USA). Partial embolization was performed using a slow
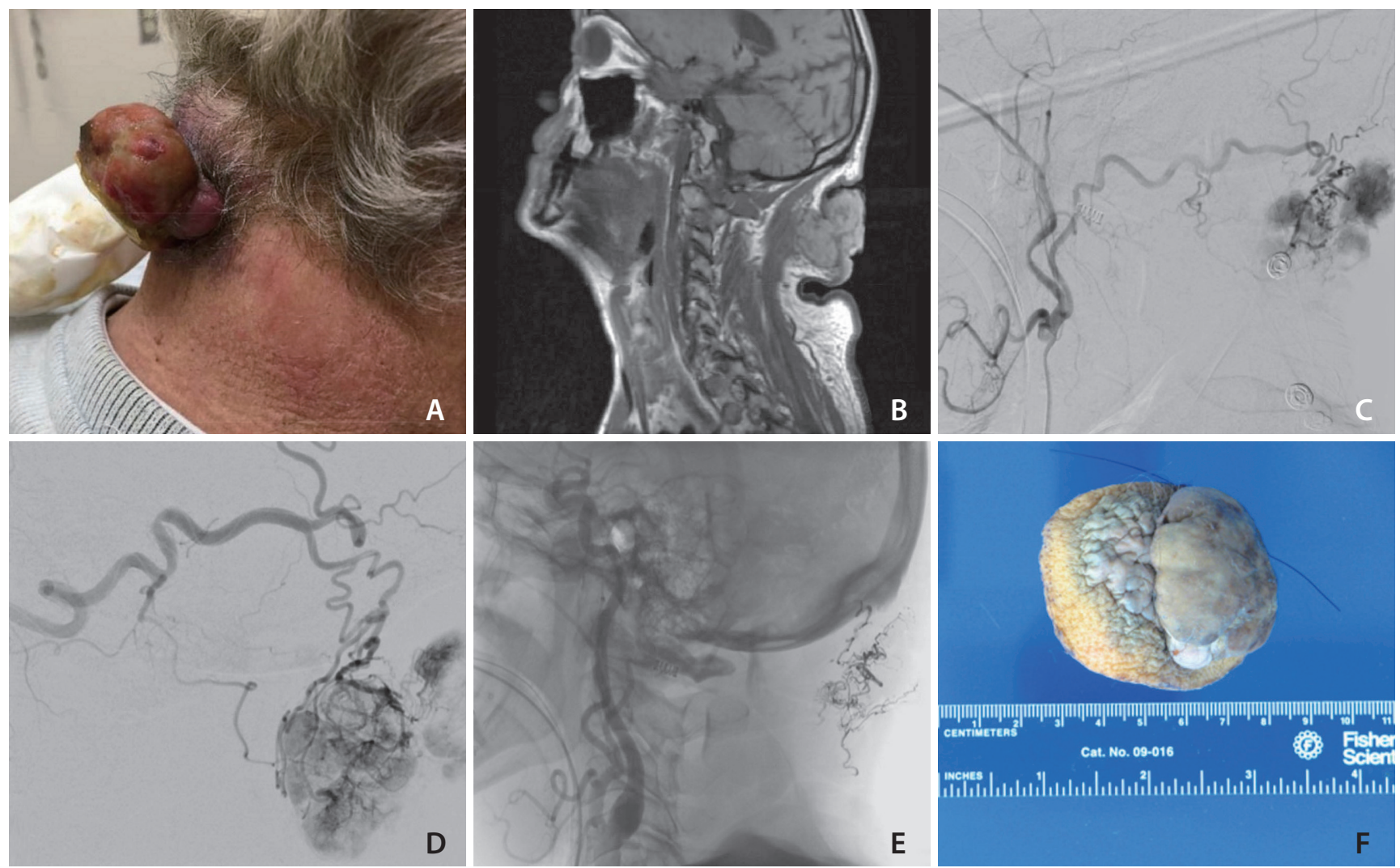

Fig. 1. (A) Photograph of the patient's posterior neck demonstrating a large, exophytic, cauliflower-shaped, grey-tan in color neck mass continuous with a yellow-tan nodule in the subcutaneous tissue measuring a $5.7 \times 5.0 \mathrm{~cm}$ with hemorrhage originating from the necrotic center. (B) T1-weighted magnetic resonance imaging of the neck revealed an exophytic, cutaneous neck mass without involvement deep to the fascia. (C) Digital subtraction angiography (DSA) of the right external carotid artery (ECA) demonstrating the primary blood supply to the mass from the right occipital artery. (D) DSA of the left ECA demonstrating the primary blood supply to the mass from the left occipital artery. (E) An unsubtracted image of the Onyx cast. (F) Gross specimen of the cauliflower shaped mass measuring approximately $5.7 \times 5.0 \mathrm{~cm}$. 
controlled injection of Onyx-18 (EV3, Irvine, CA, USA) (Fig. 1E). The large occipital artery caliber, despite its tortuosity, allowed for uncomplicated use of the balloon microcatheter to immediately halt retrograde Onyx penetration and quickly achieve tumor penetration. This same embolization sequence was performed on the contralateral side. Partial occlusion of the right and left occipital arteries was achieved with $1.31 \mathrm{~mL}$ and $0.4 \mathrm{~mL}$ of Onyx-18, respectively. Selective catheterizations were performed of the thyrocervical and costocervical trunk but no embolization was performed given the extensive reduction of the tumor's vascular supply by the occipital feeders.

The patient had an uncomplicated immediate postoperative period and reported improvement in local symptoms. Surgical resection was then performed with less than $50 \mathrm{~mL}$ of blood loss. Microscopic examination was consistent with RCC and the gross specimen demonstrated large areas of necrosis and embolization material (Fig. 1F). At one month follow up incision site was well healed and his quality of life after mass removal had improved. Due to the progression of his cancer and modest response to additional chemotherapy, he died 9 months after resection of this lesion.

\section{DISCUSSION}

RCCs account for $2 \%$ to $3 \%$ of solid malignancies in adults with $25 \%$ to $30 \%$ of patients having metastatic disease at the initial diagnosis; furthermore, about $90 \%$ of new diagnoses are discovered due to symptoms related to the metastatic disease. ${ }^{1-4}$ Cutaneous metastases from urologic malignancies are rare with a reported risk of $0.73 \%$ to $1.3 \%$, with RCC being the most common. ${ }^{5,6}$ In a retrospective series of 100,453 patients with known malignancies, a total of 77 cases had a cutaneous metastasis of which eight patients had a primary urologic malignancy. The reported incidence of cutaneous RCC metastasis range from $2.8 \%$ to $6.8 \%$, and it is thought to spread primarily via the hematogenous route secondary to the tumor's high vascularity. ${ }^{1,8,9}$

The typical hematologic pathway favors the lungs via the vena cava system, hence those with head and neck metastasis often have pulmonary disease burden as well. ${ }^{10}$ In the absence of pulmonary symptoms, dissemination via the vertebral (Batson's) venous plexus may explain those with isolated head, neck, or vertebral metastasis. ${ }^{11-13}$ Given the vascularity of RCC, the most common locations for cutaneous metasta- sis are the scalp and face since these areas are highly vascularized. ${ }^{9}$ Skin metastasis tends to be a late manifestation of the disease, usually years after nephrectomy for organ-confined disease, and associated with poor prognosis. ${ }^{6}$

Differential diagnosis for a highly vascular tumor includes angioma, metastasis, pyogenic granuloma, sweat gland tumor, angiosarcoma, Kaposi sarcoma, basal cell carcinoma, amelanotic melanoma, and sebaceous carcinoma. Skin lesions may mimic benign dermatological conditions and have patterns of nodular, inflammatory, sclerodermoid, and zosteriform findings, with nodular patterns being the most common. ${ }^{14}$

Treatment of metastatic RCC includes nephrectomy and chemotherapy (angiogenesis/multikinase inhibitors, interferon). Cutaneous lesions often require surgical removal and at times additional radiotherapy. ${ }^{1,15}$ Given the spontaneous episodes of hemorrhage and significant vascularity, our surgical colleagues requested preoperative tumor embolization. A similar treatment strategy has been performed in patients with RCC metastasis to the sinonasal cavity, especially if computed tomography or MRI demonstrates a highly vascularized tumor or in cases of spontaneous bleeding, both of which were present in our case. ${ }^{16,17}$ Preoperative embolization has been shown to provide better visibility for the surgeon, shorten operative time, reduce intraoperative blood loss while assisting tumor resection. ${ }^{16,17}$ Additionally, preoperative embolization has also been shown to reduce intraoperative blood loss in the treatment of vascular cervical spine tumors and osseous metastasis. ${ }^{2,18}$

Onyx was used in this case as its non-adhesive nature provided slower, intermittent and controlled injection, minimizing the potential risk of unintentional embolic material traveling into anastomotic vessels. In this case, bilateral occipital arteries are the major contributors of tumor vascularity and potential anastomosis into the VA and the stylomastoid branch need to be carefully monitored. ${ }^{18,19}$ Partial embolization has been shown to reduce operative blood loss and in this case was performed as a palliative measure to mitigate blood loss during resection. ${ }^{18}$ In this case, significant occlusion occurred with bilateral occipital embolization minimizing the need for further embolization.

Preoperative embolization in our case allowed successful removal of the lesion with clear margins and minimal blood loss. Although preoperative embolization of vascular tumors is common, it has not been described for treatment of a cutaneous metastatic lesion. Embolization may be a useful pre- 
operative surgical technique to aid with resection for similar presenting cutaneous vascular metastasis.

In conclusion, cutaneous RCC metastasis is rare but can present as a large hemorrhagic neck mass. Endovascular embolization followed by surgical resection is an effective method to treat highly vascularized cutaneous RCC metastasis. This description of a preoperative cutaneous head and neck tumor embolization highlights the use of primary transarterial approach embolization using a liquid embolic agent via the primary feeder despite extensive arterial supply. Cutaneous metastasis; however, have a poor prognosis and management decisions need to be individualized.

\section{Fund}

None.

\section{Ethics Statement}

Consent was obtained by patient's spouse for publication of this report.

\section{Conflicts of Interest}

The authors have no conflicts to disclose.

\section{Author Contributions}

Concept and design: MGK, LAT, VR, and MC. Analysis and interpretation: DEK. Data collection: DEK, MGK, LAT, SP, VR, and MC. Writing the article: DEK, MGK, and LAT. Critical revision of the article: DEK, MGK, LAT, SP, VR, and MC. Final approval of the article: DEK, MGK, LAT, SP, VR, and MC. Statistical analysis: none. Obtained funding: none. Overall responsibility: DEK, MGK, LAT, SP, VR, and MC.

\section{ORCID}

Dallas E. Kramer: http://orcid.org/0000-0002-2099-5864

Mena G. Kerolus: https://orcid.org/0000-0002-0902-6528

Lee A. Tan: https://orcid.org/0000-0003-3497-3321

Smita Patel: https://orcid.org/0000-0003-2866-8048

Vijaya Reddy: https://orcid.org/0000-0001-5491-552X

Michael Chen: https://orcid.org/0000-0003-3076-0214

\section{REFERENCES}

1. Porter NA, Anderson HL, Al-Dujaily S. Renal cell carcinoma presenting as a solitary cutaneous facial metastasis: case report and review of the literature. Int Semin Surg Oncol 2006;3:27
2. Sun S, Lang EV. Bone metastases from renal cell carcinoma: preoperative embolization. J Vasc Interv Radiol 1998;9:263-269

3. Weiss L, Harlos JP, Torhorst J, Gunthard B, Hartveit F, Svendsen E, et al. Metastatic patterns of renal carcinoma: an analysis of 687 necropsies. J Cancer Res Clin Oncol 1988;114:605-612

4. Gupta K, Miller JD, Li JZ, Russell MW, Charbonneau C. Epidemiologic and socioeconomic burden of metastatic renal cell carcinoma (mRCC): a literature review. Cancer Treat Rev 2008;34:193205

5. Menon AR, Thomas AS, Suresh N, Shashidhar SM. Cutaneous metastasis: an unusual presenting feature of urologic malignancies. Urol Ann 2016;8:377-380

6. Mueller TJ, Wu H, Greenberg RE, Hudes G, Topham N, Lessin SR, et al. Cutaneous metastases from genitourinary malignancies. Urology 2004;63:1021-1026

7. Saeed S, Keehn CA, Morgan MB. Cutaneous metastasis: a clinical, pathological, and immunohistochemical appraisal. J Cutan Pathol 2004:31:419-430

8. Jin WW, Chung JM, Jung KE, Park JW, Kim MH. A case of metastatic renal cell carcinoma mimicking granuloma pyogenicum. Ann Dermato/ 2008;20:263-266

9. Schwartz RA. Cutaneous metastatic disease. J Am Acad Dermatol 1995;33(2 Pt 1):161-182; quiz 183-186

10. Mahmoudi HR, Kamyab K, Daneshpazhooh M. Cutaneous metastasis of renal cell carcinoma: a case report. Dermatol Online J 2012;18:12

11. Clark L, Cossio M, Kelash F. An atypical presentation of renal cell carcinoma: isolated pleural metastases with large volume effusion. Chest 2015;148:445A

12. Sountoulides P, Metaxa L, Cindolo L. Atypical presentations and rare metastatic sites of renal cell carcinoma: a review of case reports. J Med Case Rep 2011;5:429

13. Tosco L, Palazzetti A, Crivellaro S, Guaitoli P, Abbinante M, Frea B. [Batson's paravertebral venous plexus and single vertebral metastases from renal cell carcinoma]. Urologia 2010;77 Suppl 16:42-46 Italian.

14. Brownstein MH, Helwig EB. Spread of tumors to the skin. Arch Dermatol 1973;107:80-86

15. Gay HA, Cavalieri R, Allison RR, Finley J, Quan WD. Complete response in a cutaneous facial metastatic nodule from renal cell carcinoma after hypofractionated radiotherapy. Dermatol Online J 2007;13:6

16. Bastier PL, Dunion D, de Bonnecaze G, Serrano E, de Gabory L. Renal cell carcinoma metastatic to the sinonasal cavity: a review and report of 8 cases. Ear Nose Throat J 2018;97:E6-E12

17. Pritchyk KM, Schiff BA, Newkirk KA, Krowiak E, Deeb ZE. Meta- 
static renal cell carcinoma to the head and neck. Laryngoscope 2002;112:1598-1602

18. Patsalides A, Leng LZ, Kimball D, Marcus J, Knopman J, Laufer I, et al. Preoperative catheter spinal angiography and embolization of cervical spinal tumors: outcomes from a single center.
Interv Neuroradiol 2016;22:457-465

19. Geibprasert S, Pongpech S, Armstrong D, Krings T. Dangerous extracranial-intracranial anastomoses and supply to the cranial nerves: vessels the neurointerventionalist needs to know. AJNR Am J Neuroradiol 2009;30:1459-1468 